Association for Information Systems AIS Electronic Library (AISeL)

Wirtschaftsinformatik Proceedings 2005

Wirtschaftsinformatik

February 2005

\title{
Practical Aspects of Semantic Web Technologies
}

Hans-Peter Schnurr

Ontoprise GmbH, Karlsruhe

Follow this and additional works at: http://aisel.aisnet.org/wi2005

\section{Recommended Citation}

Schnurr, Hans-Peter, "Practical Aspects of Semantic Web Technologies" (2005). Wirtschaftsinformatik Proceedings 2005. 67. http://aisel.aisnet.org/wi2005/67

This material is brought to you by the Wirtschaftsinformatik at AIS Electronic Library (AISeL). It has been accepted for inclusion in Wirtschaftsinformatik Proceedings 2005 by an authorized administrator of AIS Electronic Library (AISeL). For more information, please contact elibrary@aisnet.org. 
In: Ferstl, Otto K, u.a. (Hg) 2005. Wirtschaftsinformatik 2005: eEconomy, eGovernment, eSociety; 7. Internationale Tagung Wirtschaftsinformatik 2005. Heidelberg: Physica-Verlag

ISBN: 3-7908-1574-8

(C) Physica-Verlag Heidelberg 2005 


\title{
Practical Aspects of Semantic Web Technologies
}

\author{
Hans-Peter Schnurr \\ Ontoprise GmbH, Karlsruhe
}

\begin{abstract}
Zusammenfassung: Die Anzahl der wissenschaftlichen Veröffentlichungen, aber auch die Zahl der Forschungsprojekte im Bereich der Semantic Web Technologien hat sich innerhalb weniger Jahre stark erhöht. Das Semantic Web ist in der akademischen Welt der Informationstechnologie längst etabliert. Es drängt sich die Frage auf, welchen Status die Technologien rund um das Semantic Web in praktischen Anwendungsgebieten erreicht haben. Der Artikel beschreibt aktuelle Anwendungsgebiete in den Bereichen der semantischen Integration, innovativer Ratgeberlösungen und ontologiebasierter Suche. Zukunftsszenarien, welche durch ein Zusammenwachsen von Web Services, Sprachverarbeitung und mobilen Anwendungen mit Semantic Web Technologien möglich sind, werden dargestellt.
\end{abstract}

Schlüsselworte: Semantische Integration, ontologiebasierte Suche

\section{Im Niemandsland zwischen Forschung und Anwendung}

Die stark wachsenden Teilnehmerzahlen bei Workshops und Konferenzen rund um Semantic Web Technologien sprechen eine deutliche Sprache. Nach der dritten internationalen Semantic Web Konferenz im November 2004 in Hiroshima ${ }^{1}$ wird im Mai 2005 die zweite Europäische Semantic Web Konferenz ${ }^{2}$ stattfinden. Auch in etablierten Konferenzen rund um die Informationstechnologie füllt das Thema „Semantic Web“ die Programme. Nicht zuletzt die WI 2005 führt es als Schlagwort. Die Anzahl der wissenschaftlichen Veröffentlichungen, die Standardisierungsaktivitäten innerhalb des W3C, aber auch die Zahl der Forschungsprojekte im Bereich der Semantic Web Technologien hat sich innerhalb weniger Jahre stark erhöht. Das Semantic Web ist in der akademischen Welt der Informationstechnologie längst etabliert. Es drängt sich die Frage auf, welchen Status die Technologien rund um das Semantic Web in praktischen Anwendungsgebieten erreicht haben. Analysten der Gartner Group rechnen mit einem Durchbruch des Semantic Web im Jahr 2007. Gibt es tatsächlich eine derart große Lücke zwischen Forschung und Anwendung? Es bleibt zu klären, in welche begriffliche Schublade die aktuellen Projekte und Anwendungen fallen, die auf der Basis von Semantic Web Technologien durchgeführt werden.

\footnotetext{
http://iswc2004.semanticweb.org/

http://www.eswc2005.org/
} 


\section{Integration und Suche als Killerapplikationen}

Zunächst sind der Einsatz von Semantic Web Technologien im World Wide Web und in Unternehmen zu unterscheiden. Während die ,semantische Killerapplikation“ im WWW noch auf sich warten läßt, sind bereits heute zahlreiche Applikationen auf Basis von Semantic Web Technologien im unternehmensinternen Einsatz. Dabei werden semantische Technologien schwerpunktmäßig zur Integration heterogener Datenquellen, zur Suche, sowie zum Aufbau elektronischer Ratgebersysteme eingesetzt.

Ein Beispiel dafür ist die Enterprise Information Integration Plattform (EII) der Software AG, welche semantische Technologien der ontoprise $\mathrm{GmbH}$ enthält. Hierbei dienen Ontologien dazu, verteilte Datenquellen und Web Services einheitlich zu beschreiben und über die entsprechende Middleware inhaltlich zu integrieren.

Ein Einsatzszenario zur semantischen Suche zeigt eine Tochter der Deutschen Telekom AG, welche das DMS LiveLink ebenso an die semantische Suche angebunden hat wie das Intranet und verschiedene Datenbanken. Ziel ist es, das komplette im Unternehmen vorhandene Know-how einheitlich zu erfassen und den Nutzern zugänglich zu machen. Die Ontologien werden zur Moderation des Suchprozesses eingesetzt, damit die Anwender einfach und Kontext-gerecht auf relevante Informationen zugreifen können.

Die nahtlose Einbindung der Technologien in vorhandene Strukturen und Prozesse wird anhand eines Projektes eines Roboter-Herstellers deutlich. Dessen Servicetechniker bekommen über die mobile Anbindung des SAP Systems Aufträge des Help Desk übermittelt. Dabei werden neben den Daten der Bauteile aus dem SAP System auch Lösungsvorschläge aus einem Ratgebersystem übermittelt, welches auf Basis semantischer Technologien aufgebaut wurde und damit die Bauteiledaten mit dem Erfahrungswissen der Servicetechniker über Regeln kombiniert.

\section{Semantic Web Technologien in zukünftigen Anwendungen}

Durch das Zusammenwachsen von Web Services, mobilen Anwendungen, Sprachverarbeitungs- und Semantic Web Technologien werden auch im WWW „Killerapplikationen“ möglich. Das Forschungsprojekt „SmartWeb“3 zeigt solche Anwendungen auf. Das Fazit für praktische Aspekte von semantischen Technologien ist somit differenziert: Semantic Web Technologien bringen bereits heute im Unternehmen deutliche Mehrwerte. Die Kluft zwischen Forschung und Anwendung semantischer Technologien im WWW wird bis 2007 klar überbrückt werden.

$3 \quad$ http://smartweb.dfki.de 